\title{
INTERPOLATED-DFT-BASED FAST AND ACCURATE AMPLITUDE AND PHASE ESTIMATION FOR THE CONTROL OF POWER
}

\author{
Józef Borkowski, Dariusz Kania \\ Wroclaw University of Technology, Chair of Electronic and Photonic Metrology, B. Prusa 53/55, 50-317 Wroclaw, Poland \\ (Jozef.Borkowski@pwr.edu.pl, $\bowtie$ Dariusz.Kania@pwr.edu.pl, +4871320 6329)
}

\begin{abstract}
Quality of energy produced in renewable energy systems has to be at the high level specified by respective standards and directives. One of the most important factors affecting quality is the estimation accuracy of grid signal parameters. This paper presents a method of a very fast and accurate amplitude and phase grid signal estimation using the Fast Fourier Transform procedure and maximum decay side-lobes windows. The most important features of the method are elimination of the impact associated with the conjugate's component on the results and its straightforward implementation. Moreover, the measurement time is very short - even far less than one period of the grid signal. The influence of harmonics on the results is reduced by using a bandpass prefilter. Even using a $40 \mathrm{~dB}$ FIR pre-filter for the grid signal with $T H D \approx 38 \%, S N R \approx 53 \mathrm{~dB}$ and a $20-30 \%$ slow decay exponential drift the maximum estimation errors in a real-time DSP system for 512 samples are approximately $1 \%$ for the amplitude and approximately $8.5 \cdot 10^{-2}$ rad for the phase, respectively. The errors are smaller by several orders of magnitude with using more accurate pre-filters.
\end{abstract}

Keywords: control of power, grid signal, amplitude and phase estimation, renewable energy, interpolated DFT, maximum decay side-lobes windows.

(C) 2016 Polish Academy of Sciences. All rights reserved

\section{Introduction}

Renewable energy systems enabling production of electricity have become more and more popular in the U.S., China and EU countries in recent years [1,2]. One of the most important advantages that increase popularity of such systems is the possibility of installing them by individual users. However, to run the system smoothly (i.e., lack of congestion, high power losses, etc.) and to produce high quality energy, compliance with some standards must be assured, e.g. IEC 61727 for photovoltaic systems [3] or IEC 61400 for wind energy systems [4].

One of the most popular renewable energy systems is the photovoltaic system. It consists of three basic components: solar panels, an inverter and a control unit. The inverter is used to convert the DC input signal from the solar panels to the AC output signal, so that it is a very important part of the system. The power control process is based on constantly monitored parameters (frequency, amplitude and phase) of the grid signal. Accuracy of the parameters' estimation has a significant impact on the quality of produced energy and the system reaction time in the case of adverse events. The grid parameters determine the basis for the algorithm input of the inverter that controls switching of the internal transistors [5, 6]. Moreover, the frequency estimate is used e.g. in the FLL (Frequency Locked Loop) algorithm to synchronize the grid signal and the system output signal [7]; the amplitude estimate is used e.g. in FD (Fault Detection) algorithms [8] and in under/over voltage protection algorithms [1]; and the phase estimate is used e.g. in PLL (Phase Locked Loop) algorithms [9] and in generation of control signals in a controller [10]. 
This paper presents development of the grid signal frequency estimation method described in [11] onto an amplitude and phase estimation method using the FFT procedure and maximum decay side-lobes windows. Many estimation methods of this type enable to obtain very accurate results but in a longer time (more than 1.5 grid signal period in the measurement window) $[12,13]$. The method presented in [11] enables estimating the frequency in a short time (even far less than one period of the grid signal). The most important features of the method are: the possibility of a quick measurement, a high accuracy, straightforward implementation, minimal computational requirements, and a low cost. Moreover, this paper presents analysis of the impact of harmonics on parameter estimation errors and implementation of methods in a realtime DSP system.

An overview of the spectrum interpolation and frequency estimation is given in [11]. Currently, amplitude and phase estimation methods are also frequently developed. In [14], the author presents a method for estimation of multi-sinusoidal signal parameters that is based on a singular value decomposition method. The parameters are then used to synchronize the power grid and a distributed generation system. In [15], an estimation method for stationary and timevarying signals based on the ESPRIT method (Estimation of Signal Parameters via Rotational Invariance Technique) using AWNN (Adaptive Wavelet Neural Network) is proposed; in [16], the phase probability density function is approximated, and then the phase estimation error is minimized using the MSE (Mean Square Error). An interesting analytical solution of the phase estimation, taking into consideration the AWGN noise, is presented in [17]. The method presented in [18] is based on a type of resampling algorithm in the frequency domain A recursive technique based on the Gauss-Newton algorithm used for estimation is presented in [19]. An interesting comparison of frequency-domain and time-domain estimation algorithms is presented in [20].

The remainder of this paper is arranged as follows. Section 2 presents analytical formulas for amplitude and phase values using the FFT procedure and maximum decay side-lobes windows. In Section 3, the simulation results of amplitude and phase estimation for the pure signal and the signal in the presence of white Gaussian noise are presented. Section 4 provides the results of amplitude and phase estimation in response to changing parameter values in a real-time DSP system. Section 5 contains a study on the influence of harmonics on the estimation accuracy and the effect of the applied pre-filter on the obtained results. Section 6 presents the estimation results for the signal containing harmonics, Gaussian noise and a slow delay exponential component. Finally, the conclusions are presented in Section 7.

\section{Amplitude and phase estimation method using maximum decay side-lobes windows}

This paper presents the parameters estimation method for an $x(t)$ signal described in the time domain as follows:

$$
x(t)=\sum_{i=1}^{M} A_{i} \sin \left(2 \pi f_{i} t+\varphi_{i}\right) .
$$

Each sinusoidal oscillation is characterized by the amplitude $A_{i}$, the frequency $f_{i}$ and the phase $\varphi_{i}$. Instead of the frequency $f$, it is convenient to determine the normalized frequency $\lambda$ (also referred to as $\mathrm{CiR}$ (Cycles in Range)) with respect to the measurement time: $\lambda=f N T$ [bin], where $N$ is the number of samples of $x(t)$ signal sampled with the frequency $f_{s}=1 / T$. The frequency estimation method is presented in [11]; this paper focuses on presentation of the amplitude and phase estimation method for a sinusoidal signal.

The Discrete-time Fourier Transform (DtFT) of a signal is defined as:

$$
X(\lambda)=\sum_{n=0}^{N-1} x_{n} w_{n} e^{-j 2 \pi n \lambda / N}
$$


where: $x_{n}=x(n T) ; n=0, \ldots, N-1$ and $w_{n}$ are the time window samples. The spectrum of the primary component can be presented as the sum of the fundamental component characterized by the normalized frequency $\lambda_{1}$ and the component coupled with the frequency $-\lambda_{1}$ :

$$
X(\lambda)=\frac{A_{1}}{2 j} e^{j \varphi_{1}} W\left(\lambda-\lambda_{1}\right)-\frac{A_{1}}{2 j} e^{-j \varphi_{1}} W\left(\lambda+\lambda_{1}\right)
$$

where:

$$
W(\lambda)=\sum_{n=0}^{N-1} w_{n} e^{-j 2 \pi n \lambda / N}
$$

is the DtFT spectrum of the used time window.

In the presented estimation method, maximum decay side-lobes windows (also known as I class Rife-Vincent windows [21] or binomial coefficient windows) are used. They belong to the family of cosine windows defined as follows [21-23]:

$$
w_{n}=\sum_{h=0}^{P}(-1)^{h} a_{h} \cos \frac{2 \pi n h}{N}, n=0, \ldots, N-1,
$$

where: $P=H-1$ defines the number of used cosine functions; $H$ is the window order, and $a_{h}$ are the window coefficients. The windows used in the method have maximum decay amplitudes of side-lobes from all cosine windows.

The spectrum of the maximum decay side-lobes windows can be approximated for $H>1$, $\lambda \ll N, N » 1$ by [23]:

$$
W(\lambda)=\frac{D(\lambda)}{P(\lambda)}
$$

where:

$$
\begin{gathered}
D(\lambda)=\frac{N(2 H-2) !}{\pi 2^{2 H-2}} \sin (\pi \lambda) e^{-j \pi \lambda}, \\
P(\lambda)=\lambda \prod_{h=1}^{H-1}\left(h^{2}-\lambda^{2}\right)=\frac{\prod_{h=0}^{H}\left(h^{2}-\lambda^{2}\right)}{(-\lambda)(H-\lambda)(H+\lambda)} .
\end{gathered}
$$

The values of coefficients $a_{h}$ for windows with $H \leq 7$, together with the most important parameters, can be found in [11].

The method given in [11] enables determining the normalized frequency $\lambda_{1}$. It is based on three relevant consecutive points of the spectrum $X(\lambda)$ for $\lambda=k-1, k, k+1$ (values $X_{k-1}, X_{k}$, $X_{k+1}$ ) around the main lobe, where $k$ is the index of DFT spectrum $X_{k}$, i.e. (2) for integer $\lambda$. An important advantage of the method is taking into consideration the effect of a coupled component having the frequency $-\lambda_{1}$.

The equations for the amplitude and phase can be determined analytically. Based on (7) and (8), they can be written for $\lambda=k \pm \lambda_{1}$ :

$$
D\left(k \pm \lambda_{1}\right)=\frac{N(2 H-2) !}{\pi 2^{2 H-2}} \sin \left(\pi\left(k \pm \lambda_{1}\right)\right) e^{-j \pi\left(k \pm \lambda_{1}\right)}
$$

and

$$
P\left(k \pm \lambda_{1}\right)=\frac{\prod_{h=0}^{H}\left(h^{2}-\left(k \pm \lambda_{1}\right)^{2}\right)}{\left(-\left(k \pm \lambda_{1}\right)\right)\left(H-\left(k \pm \lambda_{1}\right)\right)\left(H+\left(k \pm \lambda_{1}\right)\right)} .
$$


Introducing the following notation:

$$
\begin{gathered}
F^{-}=\frac{A_{1}}{2 j} e^{j \varphi_{1}} D\left(k-\lambda_{1}\right), \\
F^{+}=-\frac{A_{1}}{2 j} e^{-j \varphi_{1}} D\left(k+\lambda_{1}\right),
\end{gathered}
$$

taking into account that:

$$
D\left(k-1 \pm \lambda_{1}\right)=D\left(k \pm \lambda_{1}\right)=D\left(k+1 \pm \lambda_{1}\right)
$$

and (6) and (3) for $\lambda=k-1, k, k+1$, three equations are obtained:

$$
\left\{\begin{array}{c}
\frac{F^{+}}{P\left(k-1+\lambda_{1}\right)}+\frac{F^{-}}{P\left(k-1-\lambda_{1}\right)}-X_{k-1}=0 \\
\frac{F^{+}}{P\left(k+\lambda_{1}\right)}+\frac{F^{-}}{P\left(k-\lambda_{1}\right)}-X_{k}=0 \\
\frac{F^{+}}{P\left(k+1+\lambda_{1}\right)}+\frac{F^{-}}{P\left(k+1-\lambda_{1}\right)}-X_{k+1}=0
\end{array},\right.
$$

where:

$$
\begin{aligned}
& P\left(k-1 \pm \lambda_{1}\right)=-\frac{\left(H-k \mp \lambda_{1}\right)}{H-1+k \pm \lambda_{1}} P\left(k \pm \lambda_{1}\right), \\
& P\left(k+1 \pm \lambda_{1}\right)=-\frac{\left(H+k \pm \lambda_{1}\right)}{H-1-k \mp \lambda_{1}} P\left(k \pm \lambda_{1}\right) .
\end{aligned}
$$

Based on (11) and (12), the amplitude estimate $\hat{A}_{1}$ can be obtained, which is burdened with some error (because of the assumptions $\lambda \ll N, N » 1$ and real signal distortions):

$$
\left\{\begin{array}{c}
\hat{A}_{1}=\frac{2 j F^{-}}{e^{j \varphi_{1}} D\left(k-\lambda_{1}\right)}=\frac{e^{-j \varphi_{1}} 2 j F^{-}}{D\left(k-\lambda_{1}\right)} \\
\hat{A}_{1}=-\frac{2 j F^{+}}{e^{-j \varphi_{1}} D\left(k+\lambda_{1}\right)}
\end{array} \Rightarrow \hat{A}_{1}=2 \sqrt{\frac{F^{-} F^{+}}{D\left(k+\lambda_{1}\right) D\left(k-\lambda_{1}\right)}},\right.
$$

where $F^{+}$and $F^{-}$can be obtained after transformation (14), taking into account two points of the signal spectrum. For example, for points $X_{k-1}$ and $X_{k}$ :

$$
\begin{gathered}
F^{+}=\frac{P\left(k-1-\lambda_{1}\right) P\left(k+\lambda_{1}\right)}{P\left(k-1-\lambda_{1}\right) P\left(k+\lambda_{1}\right)-P\left(k-1+\lambda_{1}\right) P\left(k-\lambda_{1}\right)} \cdot\left(X_{k} P\left(k-\lambda_{1}\right)-X_{k-1} \frac{P\left(k-1+\lambda_{1}\right) P\left(k-\lambda_{1}\right)}{P\left(k+\lambda_{1}\right)}\right), \\
F^{+}=\frac{P\left(k-1-\lambda_{1}\right) P\left(k+\lambda_{1}\right)}{P\left(k-1-\lambda_{1}\right) P\left(k+\lambda_{1}\right)-P\left(k-1+\lambda_{1}\right) P\left(k-\lambda_{1}\right)} \cdot\left(X_{k-1} P\left(k-1+\lambda_{1}\right)-X_{k} \frac{P\left(k-1+\lambda_{1}\right) P\left(k-\lambda_{1}\right)}{P\left(k-1+\lambda_{1}\right)}\right) .
\end{gathered}
$$

Substituting (18) and (19) in (17), the following is obtained for $k=1$ and $H=2$ :

$$
\hat{A}_{1}=2 \frac{e^{j \lambda_{1} \pi}\left(1+\lambda_{1}\right)}{3 N \operatorname{sinc}\left(\left(\lambda_{1}-1\right) \pi\right)} \cdot \sqrt{-\left(\lambda_{1}^{2}-4\right)\left(X_{1}\left(\lambda_{1}+2\right)-X_{0}\left(\lambda_{1}-1\right)\right)\left(X_{1}\left(\lambda_{1}-2\right)-X_{0}\left(\lambda_{1}+1\right)\right)} .
$$


The value of signal phase can be estimated based on (11) and (12) from:

$$
\hat{\varphi}_{1}=\arg \left(\frac{2 j F^{-}}{\hat{A}_{1} D\left(k-\lambda_{1}\right)}\right)
$$

or

$$
\hat{\varphi}_{1}=\arg \left(-\frac{\hat{A}_{1} D\left(k+\lambda_{1}\right)}{2 j F^{+}}\right) .
$$

For the spectrum points $X_{k-1}$ and $X_{k},(21)$ for $k=1$ and $H=2$ is:

$$
\hat{\varphi}_{1}=\arg \left(-\frac{2 j e^{j \pi\left(\lambda_{1}-1\right)}\left(\lambda_{1}-2\right)}{\hat{A}_{1} N \operatorname{sinc}\left(\left(\lambda_{1}-1\right) \pi\right)\left(\lambda_{1}+2+2\left(\lambda_{1}-1\right)\right)} \cdot\left(\lambda_{1} X_{1}\left(\lambda_{1}+1\right)\left(\lambda_{1}+2\right)-\lambda_{1} X_{0}\left(\lambda_{1}^{2}-1\right)\right)\right) .
$$

\section{Simulation research}

Simulation research of the estimation method was performed in the MATLAB environment. There was used the Hanning window $(H=2)$, which has a good suppression of side-lobes and a small width of the main lobe. Research included analysis of the impact of measurement parameters on the accuracy of amplitude and phase estimation for the pure sinusoidal signal and for the signal disturbed by Gaussian white noise. The signal frequency was known a priori, but, for practical application, it can be obtained using the method presented in [11].

First, the impact of the number of samples $N$ on the systematic errors of amplitude and phase estimation was examined for the pure sinusoidal signal. The signal phase $\varphi_{1}$ was changed from 0 to $2 \pi$ in steps of $0.01 \mathrm{rad}$. For each $C i R$ value, the maximum error of the entire range of $\varphi_{1}$ was taken. Values of $N$ were changed in the range of $2^{5}, \ldots, 2^{11}$ (because of the FFT radix-2 algorithm). The value of $k$ was 1 because of the range of tested $C i R$ values $(0.1<C i R<2)$.

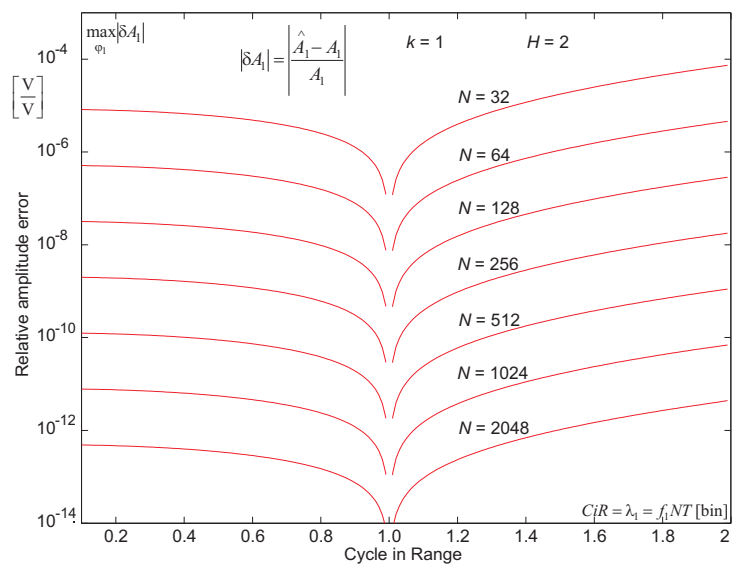

Fig. 1. The systematic amplitude error $\delta A_{1}$ for the method in Section $2:\left|\delta A_{1}\right|$ is inversely proportional to $N^{4}$, the smallest error is for $C i R=1$. 


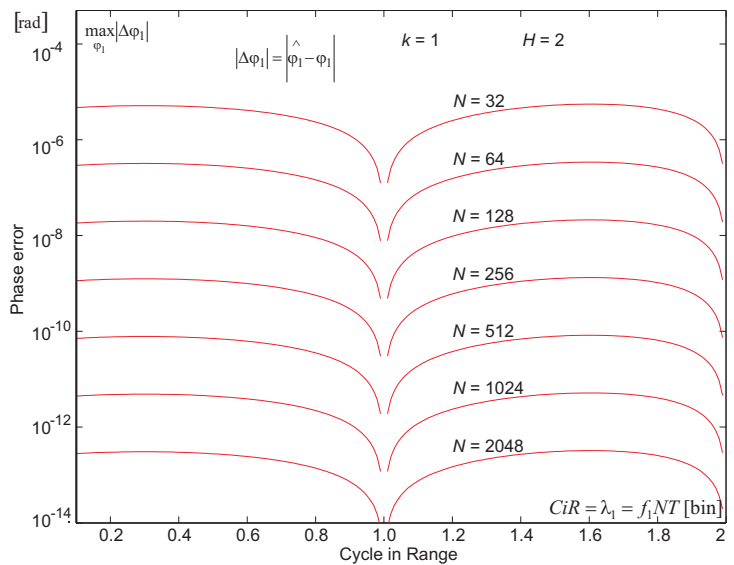

Fig. 2. The systematic phase error $\delta \varphi_{1}$ for the method in Section $2:\left|\delta \varphi_{1}\right|$ is inversely proportional to $N^{4}$; the smallest error is for $C i R=1$.

The systematic error of amplitude and phase estimation method (for the worst phase case) is inversely proportional to $N^{4}$ (Figs. 1,2). Increasing the $N$ value at the constant $C i R$ value increases the estimation accuracy. The smallest estimation error (global minimum) occurs for $C i R=1-$ when there is one period of the signal in the measurement window. Figs. 1 and 2 differ by the fact that in Fig. 1 there is no local minimum for $C i R=2$, whereas in Fig. 2 it exists, due to the properties of the method for $H=2$.

The Gaussian white noise that was subsequently added to the signal in the second stage of research was generated using the $\operatorname{randn}()$ function. The estimation results were compared with corresponding Cramer-Rao bounds (Figs. 3, 4) [24]. The variance estimator was, in this case, eMSE (Empirical Mean Square Error) assuming that the systematic square error is negligibly small in relation to the variance estimator. The upper value of the SNR (Signal to Noise Ratio) range corresponds to the actual dynamics of the 16-bit A/D converter. The number of realization was $10^{5}$, the phase $\varphi_{1}$ was changed from 0 to $2 \pi$ in steps of $0.01 \mathrm{rad}$ and the value of $N$ was 512 .

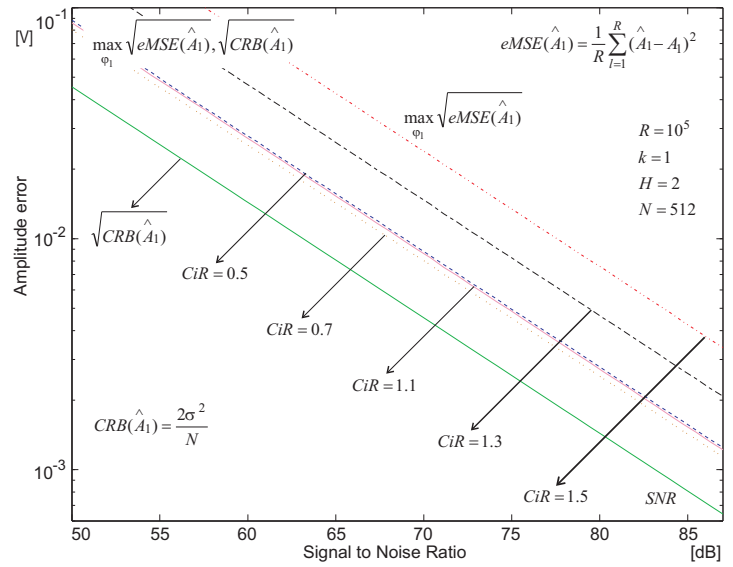

Fig. 3. The statistical properties of the proposed method for estimating the $A_{1}$ amplitude: the $e M S E$ error and the Cramer-Rao bound as a function of $S N R$ and for sample $C i R$ values. 


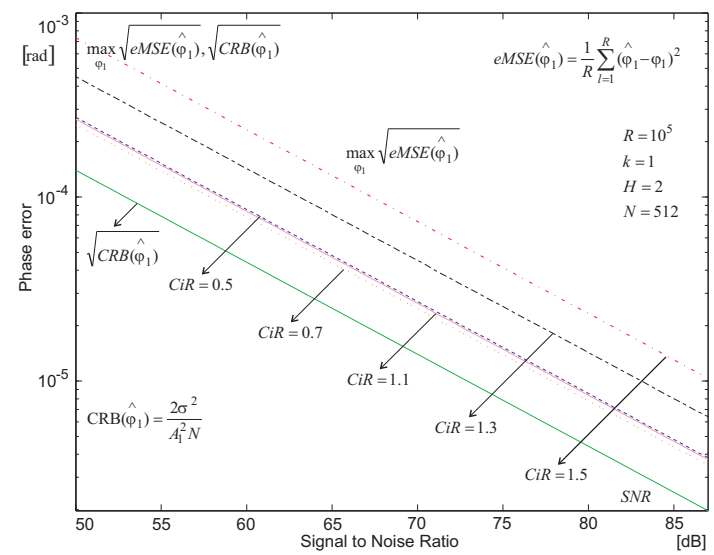

Fig. 4. The statistical properties of the proposed method for estimating the $\varphi_{1}$ phase: the $e M S E$ error and the Cramer-Rao bound as a function of $S N R$ and for sample $C i R$ values.

For a given $C i R$ value, increasing the $S N R$ value (decreasing the noise power $\sigma^{2}$ added to the signal) decreases estimation errors. The square root of $e M S E$ errors to the $C R B$ bounds was constant regardless of the $S N R$ value (Figs. 3, 4). The ratio value ranged from approximately 1.76 for $C i R=0.7$ to approximately 5.25 for $C i R=1.5$. For $C i R<1$ errors decrease and for $\mathrm{CiR}$ $>1$ errors increase due to the properties of the method for $H=2$.

\section{Real time implementation in DSP system and reaction time to changing parameters}

To confirm correct functioning of the estimation method and accuracy of the obtained results, the method was implemented in a real-time DSP system with the same hardware structure as presented in [11] (Fig. 5) and the software extension to estimate the amplitude (20) and the phase (23). The test analogue signal $x(t)$ was generated using an Arbitrary Waveform Generator (AWG) with a 14-bit D/A converter. To convert the $x(t)$ signal into the digital form, a 16-bit A/D converter at $24 \mathrm{kHz}$ sampling rate was used. Processing of the measurement data was performed using a TMS320C6713 floating-point Digital Signal Processor (DSP) with $225 \mathrm{MHz}$ clock rate. The last $N$ samples of the signal were stored in the DSP memory because they were needed to calculate the current estimate. The frequency, amplitude and phase values were updated every four sampling periods (167 $\mu$ s).

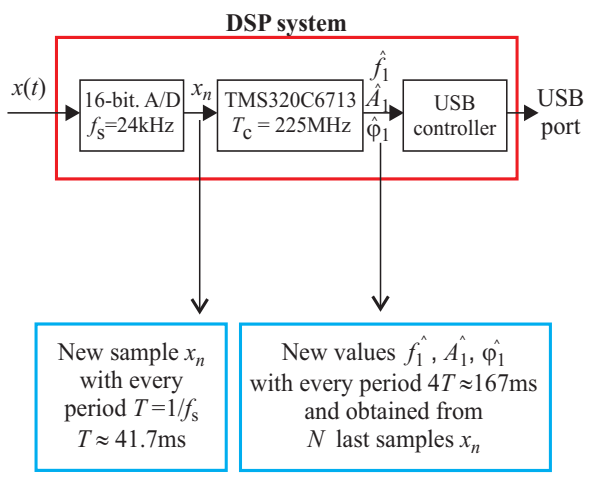

Fig. 5. The real-time measurement system for signal parameter estimation. 
Test signals were generated as recommended by the IEEE Standard for Synchrophasors for Power Systems [25]. They can also be e.g. test signals used for synchronization between the power grid and the distributed generation system [26]. In the case of amplitude estimation, the effect of amplitude's $10 \%$ jump at $t=0$ was examined (Fig. 6a), whereas, in the case of phase estimation, the effect of phase $90^{\circ}$ jump at $t=0$ was examined (Fig. 6b).

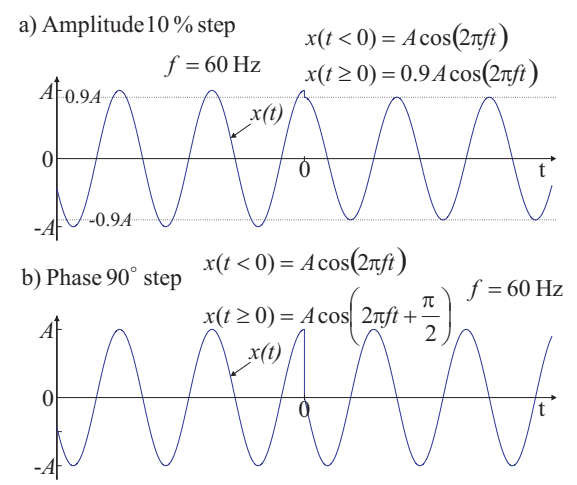

Fig. 6. Test signals generated in the DSP system: a) the test signal for amplitude estimation; b) the test signal for phase estimation.

A sudden jump in the estimated value causes appearing so-called transient states. The greater the $N$ value at the constant sampling frequency (greater $C i R$ value), the longer the transient states (Figs. 7, 8). The time required for amplitude estimation with an accuracy of $1 \%$ was ca. $2.16 \cdot 10^{-3} \mathrm{~s}$ (ca. $\left.0.81 N T\right)$ for $C i R \approx 0.16$, ca. $4.38 \cdot 10^{-3} \mathrm{~s}$ (ca. $0.82 N T$ ) for $C i R \approx 0.32$, and ca. $8.38 \cdot 10^{-3} \mathrm{~s}$ (ca. $0.78 \mathrm{NT}$ ) for $C i R \approx 0.64$. The maximum relative estimation errors for amplitude estimation in the steady state were as follows: for $N=64$ the error was ca. $3.1 \cdot 10^{-3}$; for $N=128$ - ca. $2.5 \cdot 10^{-3}$; and for $N=256$ - ca. $1.8 \cdot 10^{-3}$. In the case of phase estimation, the time required to obtain results with an accuracy of $0.15 \mathrm{rad}$ was ca. $1.39 \cdot 10^{-3} \mathrm{~s}(\mathrm{ca} .0 .52 \mathrm{NT}$ ) for $C i R \approx 0.16$, ca. $3.27 \cdot 10^{-3} \mathrm{~s}$ (ca. $0.61 \mathrm{NT}$ ) for $C i R \approx 0.32$, and ca. $7.59 \cdot 10^{-3} \mathrm{~s}$ (ca. $0.71 \mathrm{NT}$ ) for $C i R \approx 0.64$ (Fig. 8). The maximum estimation errors in the steady state were as follows: for $N=64$, the error was ca. $7.4 \cdot 10^{-2} \mathrm{rad}$; for $N=128$ - ca. $6.5 \cdot 10^{-2} \mathrm{rad}$; and for $N=256-\mathrm{ca} .5 .5 \cdot 10^{-2} \mathrm{rad}$. The smaller the value of $\mathrm{CiR}$, the higher the phase spike in the transient state. The highest spike was for $N=64$, but it does not exceed $10 \%$ of the actual phase value.

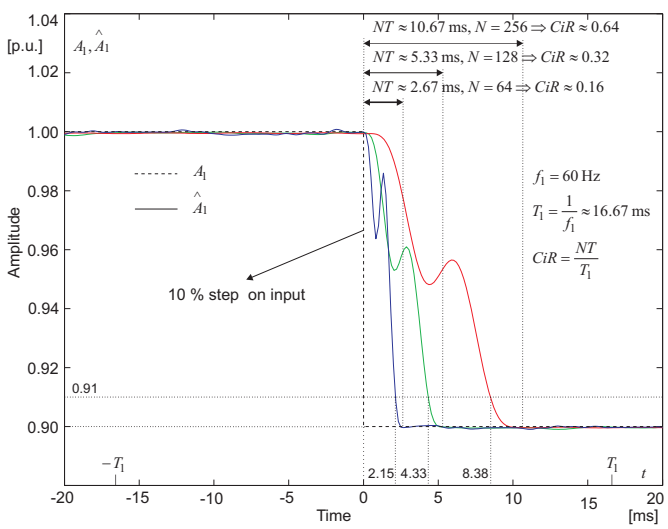

Fig. 7. The effect of $10 \%$ amplitude jump of the tested signal in the amplitude estimation using the proposed method for $N=64,128,256$ : the transient time is longer when $N$ is greater, and $1 \%$ accuracy is obtained after ca. $0.8 N T$. 


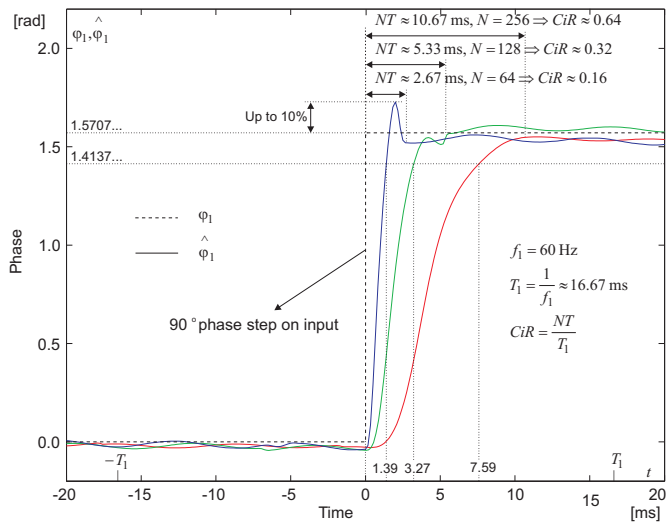

Fig. 8. The effect of $90^{\circ}$ phase jump of the tested signal in the phase estimation using the proposed method for $N=64,128,256$ : the transient time is longer when $N$ is greater, and 0.15 rad accuracy is obtained after ca. $0.5 N T$ for $N=64$, ca. $0.6 N T$ for $N=128$, and ca. $0.7 N T$ for $N=256$.

\section{Influence of harmonics and necessity of pre-filtering}

In practice, the grid signal is not a pure sinusoid. It can be distorted in a deterministic way by harmonics or in a random way by white, colored or quantization noise. The presence of harmonics in the grid signal decreases the energy quality. They can have various sources, e.g. working of non-linear loads. The grid signal spectrum contains, except the component (3) corresponding to the grid frequency $f_{1}$, additional components of a form similar to (3) but with parameters $A_{i}, f_{i}, \varphi_{i}$, where $f_{i}=i \cdot f_{1}, i=2,3, \ldots, M$. In practice, the first seven harmonics $(M=7)$ have the greatest impact, and their amplitudes do not exceed $10 \%$ of the fundamental component [27].

In the simulation research, the effect of the 2 nd to the 7 th harmonics was determined (Tables 1,2$)$ for the cases of the tested signal containing only one harmonic (columns for $i=2, \ldots, 7$ in Tables 1,2$)$ and simultaneously containing 2 or 3 harmonics: $i=2,3 ; i=3,4$, and $i=2,3,4$ (the last three columns in Tables 1,2 ). The amplitudes of harmonics were equal to $10 \%$ of the fundamental component, and the estimation error was determined as the maximum amplitude estimation error (Table 1) and as the maximum phase error (Table 2). The phase $\varphi_{1}$ of the fundamental component was changed in the full range ( 0 to $2 \pi$ in steps of $0.01 \mathrm{rad})$, which is necessary because the $\varphi_{1}$ value has a significant impact on the frequency estimation error [11] as well as the amplitude and phase estimation errors. It is necessary to pre-filter the signal to significantly increase the accuracy of $A_{1}$ and $\varphi_{1}$ estimation. Tables 1,2 show the maximum estimation error (for the worst phase $\varphi_{1}$ case) for two cases: 1 ) without pre-filtration and 2) using two arbitrarily chosen band-pass FIR filters marked A (with $40 \mathrm{~dB}$ band-stop attenuation) and $\mathrm{B}$ (with $60 \mathrm{~dB}$ band-stop attenuation). The use of band-pass filter enables limiting the influence of harmonics and slow damped disturbances (including the constant component).

The results presented in Tables 1, 2 show that estimation errors are strongly dependent on the applied filters and that the use of A filter enables achieving accurate estimations of amplitude $A_{1}$ (the error below 1\%) and phase $\varphi_{1}$ (the error below $0.001 \mathrm{rad}$ ). The use of better filters (e.g. B) improves the estimation accuracy. The limitations are the maximum error values presented for the pure sinusoid (Figs. 1, 2). However, a better filter requires a faster processor in real-time execution, which usually increases the system response time.

The results in Tables 1, 2 show also a typical dependence of estimation errors on the $\mathrm{CiR}$ value (the measurement time). In analogy to that of $f_{1}$ estimation [11], the $A_{1}$ and $\varphi_{1}$ estimation 
errors usually decrease when the $C i R$ increases in the tested range $C i R=0.13, \ldots, 1.07$, such as for the undistorted signal (Figs. 1, 2). For $\varphi_{1}$ estimation this effect can be observed more clearly than for $A_{1}$ estimation. Additionally, when increasing the harmonic number $i$, the $\varphi_{1}$ estimation error usually decreases (Table 2). The influence of inter-harmonics is similar because there are no significant restrictions for $f_{i}$. In Tables 1 and $2 f_{i}=i \cdot f_{1}$ because of its greatest significance for the purpose of research.

Table 1. The influence of the 2 nd to 7 th harmonics on the results of basic component amplitude estimation without and with using two band-pass FIR pre-filters: A \& B.

\begin{tabular}{|c|c|c|c|c|c|c|c|c|c|c|}
\hline \multirow[t]{2}{*}{ CiR } & \multirow{2}{*}{$\begin{array}{l}\text { Filter }{ }^{1)} \\
\left.\text { for }^{2}\right) \\
i=\end{array}$} & \multicolumn{9}{|c|}{ Amplitude estimation error [\%] } \\
\hline & & 2 & 3 & 4 & 5 & 6 & 7 & $2 \& 3$ & $3 \& 4$ & $2 \& 3 \& 4$ \\
\hline \multirow{3}{*}{0.13} & no filter & 15 & 12 & 9.4 & 22 & 38 & 48 & 27 & 19 & 31 \\
\hline & filter $A$ & 0.47 & 0.43 & 0.48 & 0.48 & 0.67 & 0.57 & 0.49 & 0.47 & 0.51 \\
\hline & filter B & 0.041 & 0.045 & 0.041 & 0.041 & 0.059 & 0.066 & 0.047 & 0.046 & 0.048 \\
\hline \multirow{3}{*}{0.27} & no filter & 8.9 & 20 & 25 & 13 & 7.6 & 14 & 25 & 45 & 48 \\
\hline & filter $A$ & 0.45 & 0.44 & 0.59 & 0.45 & 0.46 & 0.46 & 0.46 & 0.56 & 0.56 \\
\hline & filter B & 0.039 & 0.049 & 0.046 & 0.039 & 0.041 & 0.046 & 0.051 & 0.058 & 0.059 \\
\hline \multirow{3}{*}{0.53} & no filter & 14 & 4.1 & 5.1 & 6.4 & 2.3 & 1.1 & 11 & 8.8 & 5.9 \\
\hline & filter $A$ & 0.47 & 0.42 & 0.45 & 0.42 & 0.42 & 0.42 & 0.46 & 0.45 & 0.5 \\
\hline & filter $B$ & 0.039 & 0.039 & 0.039 & 0.037 & 0.037 & 0.037 & 0.039 & 0.041 & 0.039 \\
\hline \multirow{3}{*}{1.07} & no filter & 3.6 & 0.18 & 0.065 & 0.038 & 0.025 & 0.017 & 3.8 & 1.9 & 3.8 \\
\hline & filter $A$ & 0.43 & 0.42 & 0.42 & 0.42 & 0.42 & 0.42 & 0.43 & 0.42 & 0.43 \\
\hline & filter B & 0.037 & 0.037 & 0.037 & 0.037 & 0.037 & 0.037 & 0.038 & 0.037 & 0.038 \\
\hline
\end{tabular}

1) Filter A \& B: $F_{\text {stop } 1}=10 \mathrm{~Hz}, \mathrm{~F}_{\text {pass } 1}=40 \mathrm{~Hz}, \mathrm{~F}_{\text {pass } 2}=60 \mathrm{~Hz}, \mathrm{~F}_{\text {stop } 2}=90 \mathrm{~Hz}$;

filter $\mathrm{A}: \mathrm{A}_{\text {stop } 1}=40 \mathrm{~dB}, \mathrm{~A}_{\mathrm{stop} 2}=40 \mathrm{~dB}, \mathrm{~A}_{\text {pass }}=0.1 \mathrm{~dB}$, order 1686 ;

filter B: $A_{\text {stop } 1}=60 \mathrm{~dB}, A_{\text {stop } 2}=60 \mathrm{~dB}, A_{\text {pass }}=0.01 \mathrm{~dB}$. order 2738 .

2) All harmonics have amplitude eq. to $10 \%$ of the basic component, and the phase eq. 0 rad with respect to the basic component.

Table 2. The influence of the 2 nd to 7 th harmonics on the results of basic component phase estimation with the FIR pre-filters: A \& B.

\begin{tabular}{|c|c|c|c|c|c|c|c|c|c|c|}
\hline \multirow[t]{2}{*}{ CiR } & \multirow{2}{*}{$\begin{array}{c}\text { Filter }^{1)} \\
\left(\mathrm{for}^{2)}\right) \\
i=\end{array}$} & \multicolumn{9}{|c|}{ Phase estimation error [rad] } \\
\hline & & 2 & 3 & 4 & 5 & 6 & 7 & $2 \& 3$ & $3 \& 4$ & $2 \& 3 \& 4$ \\
\hline \multirow{3}{*}{0.13} & no filter & $1.5 \times 10^{-1}$ & $1.3 \times 10^{-1}$ & $9.5 \times 10^{-2}$ & $2.3 \times 10^{-1}$ & $3.9 \times 10^{-1}$ & $5.1 \times 10^{-1}$ & $2.8 \times 10^{-1}$ & $1.8 \times 10^{-1}$ & $3.2 \times 10^{-1}$ \\
\hline & filter $\mathrm{A}$ & $5.5 \times 10^{-4}$ & $1.6 \times 10^{-4}$ & $6.3 \times 10^{-4}$ & $6.4 \times 10^{-4}$ & $2.5 \times 10^{-3}$ & $1.6 \times 10^{-3}$ & $7.1 \times 10^{-4}$ & $5.9 \times 10^{-4}$ & $8.7 \times 10^{-4}$ \\
\hline & filter B & $2.9 \times 10^{-5}$ & $7.6 \times 10^{-5}$ & $3.3 \times 10^{-5}$ & $3.1 \times 10^{-5}$ & $2.2 \times 10^{-4}$ & $2.9 \times 10^{-4}$ & $1.1 \times 10^{-4}$ & $9.3 \times 10^{-5}$ & $1.2 \times 10^{-4}$ \\
\hline \multirow{3}{*}{0.27} & no filter & $8.9 \times 10^{-2}$ & $2.0 \times 10^{-1}$ & $2.6 \times 10^{-1}$ & $1.3 \times 10^{-1}$ & $7.3 \times 10^{-2}$ & $1.4 \times 10^{-1}$ & $2.5 \times 10^{-1}$ & $4.6 \times 10^{-1}$ & $5.0 \times 10^{-1}$ \\
\hline & filter $A$ & $3.2 \times 10^{-4}$ & $2.6 \times 10^{-4}$ & $1.7 \times 10^{-3}$ & $3.6 \times 10^{-4}$ & $4.3 \times 10^{-4}$ & $4.6 \times 10^{-4}$ & $4.9 \times 10^{-4}$ & $1.5 \times 10^{-3}$ & $1.6 \times 10^{-3}$ \\
\hline & filter B & $1.7 \times 10^{-5}$ & $1.2 \times 10^{-4}$ & $9.2 \times 10^{-5}$ & $1.8 \times 10^{-5}$ & $3.8 \times 10^{-5}$ & $8.6 \times 10^{-5}$ & $1.3 \times 10^{-4}$ & $2.1 \times 10^{-4}$ & $2.2 \times 10^{-4}$ \\
\hline \multirow{3}{*}{0.53} & no filter & $1.4 \times 10^{-1}$ & $4.0 \times 10^{-2}$ & $5.0 \times 10^{-2}$ & $6.4 \times 10^{-3}$ & $2.2 \times 10^{-3}$ & $1.1 \times 10^{-3}$ & $1.1 \times 10^{-1}$ & $8.7 \times 10^{-2}$ & $6.1 \times 10^{-2}$ \\
\hline & filter $A$ & $5.1 \times 10^{-4}$ & $4.8 \times 10^{-5}$ & $3.5 \times 10^{-4}$ & $1.8 \times 10^{-5}$ & $1.5 \times 10^{-5}$ & $3.6 \times 10^{-6}$ & $4.6 \times 10^{-4}$ & $3.1 \times 10^{-4}$ & $8.1 \times 10^{-4}$ \\
\hline & filter $B$ & $2.6 \times 10^{-5}$ & $2.3 \times 10^{-5}$ & $1.8 \times 10^{-5}$ & $9.1 \times 10^{-7}$ & $1.3 \times 10^{-6}$ & $6.8 \times 10^{-7}$ & $1.2 \times 10^{-5}$ & $4.1 \times 10^{-5}$ & $1.7 \times 10^{-5}$ \\
\hline \multirow{3}{*}{1.07} & no filter & $3.6 \times 10^{-2}$ & $1.8 \times 10^{-3}$ & $6.5 \times 10^{-4}$ & $3.8 \times 10^{-4}$ & $2.5 \times 10^{-4}$ & $1.7 \times 10^{-4}$ & $3.8 \times 10^{-2}$ & $2.4 \times 10^{-3}$ & $3.8 \times 10^{-2}$ \\
\hline & filter $A$ & $1.3 \times 10^{-4}$ & $2.3 \times 10^{-6}$ & $4.5 \times 10^{-6}$ & $1.1 \times 10^{-6}$ & $1.6 \times 10^{-6}$ & $5.5 \times 10^{-7}$ & $1.3 \times 10^{-4}$ & $2.6 \times 10^{-6}$ & $1.3 \times 10^{-4}$ \\
\hline & filter B & $6.9 \times 10^{-6}$ & $1.1 \times 10^{-6}$ & $2.4 \times 10^{-7}$ & $5.4 \times 10^{-8}$ & $1.4 \times 10^{-7}$ & $1.1 \times 10^{-7}$ & $7.9 \times 10^{-6}$ & $1.3 \times 10^{-6}$ & $8.1 \times 10^{-6}$ \\
\hline
\end{tabular}

\section{Real-time implementation with pre-filtering}

To experimentally verify effectiveness of the proposed method for estimation of the grid signal parameters, the DSP system (Fig. 5) was supplemented in the software layer with prefiltration using a bandpass FIR filter corresponding to A filter in Section 5. The test signal $x(t)$ with frequency $f_{1}=50 \mathrm{~Hz}\left(\omega=2 \pi f_{1}\right)$ was distorted in a significant way by four harmonics 
(THD $\approx 38 \%$ ), white noise with the zero expected value and the standard deviation $\sigma=0.05$ (that means $S N R \approx 53 \mathrm{~dB}$ for the $50 \mathrm{~Hz}$ component), as well as the exponential component with parameters typical for an industrial load [28-31] (Fig. 9). The test signal was generated using an Arbitrary Waveform Generator (AWG) with a 14-bit D/A converter. For $N=512$ $(C i R \approx 1.07)$ and $N=256(C i R \approx 0.53)$, the signal frequency was estimated by the method described in [11], then the amplitude was estimated from (20), and finally the signal phase was estimated from (23). To eliminate harmonics, the DC offset and slow decay exponential drift of the bandpass FIR filter (A filter) were applied before estimation. The lower the $C i R$ value, the shorter the total response time of the estimation method with filtering (Fig. 10).
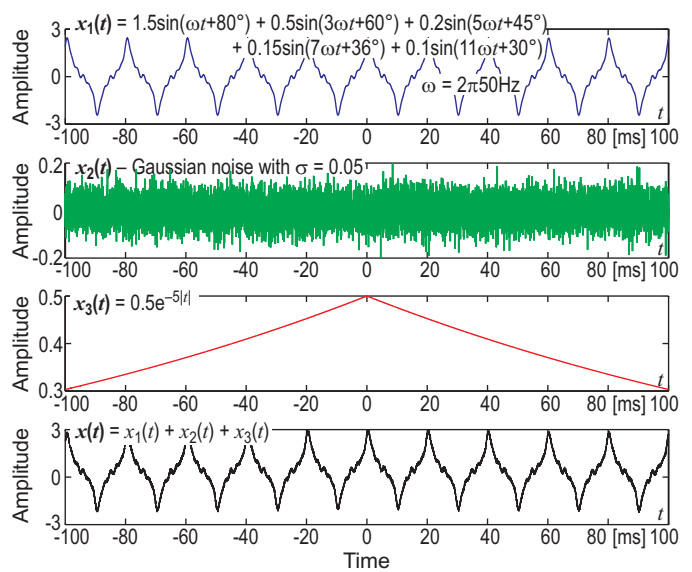

Fig. 9. The grid signal $x(t)$, tested in the final experiment, as the sum of $x_{1}(t)-50 \mathrm{~Hz}$ sinusoid with four harmonics, $x_{2}(t)$ - Gaussian noise, and $x_{3}(t)$ - exponential component. All parameter values are the same as in [28-31] to make possible comparison of the results.

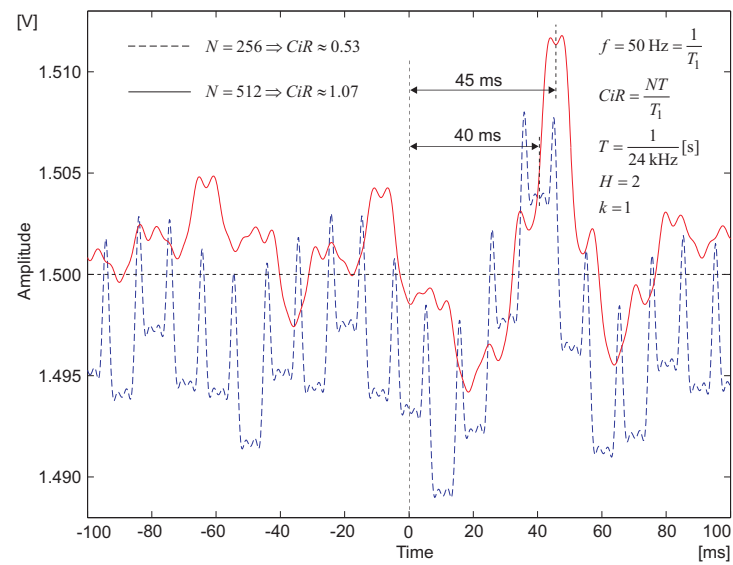

Fig. 10. The total delay of the proposed estimation method in the example of amplitude estimation: the marked times, $45 \mathrm{~ms}$ (for $\mathrm{CiR} \approx 1.07$ ) and $40 \mathrm{~ms}$ (for $C i R \approx 0.53$ ), show the delay effect of the exponential part $x_{3}(t)$ on its maximum value ( $t=0$ and Fig. 9).

For $N=512(C i R \approx 1.07)$, the maximum amplitude estimation error was ca. $0.49 \%$ without the exponential component and ca. $0.91 \%$ after the occurrence of this component, whereas, for $N=256(C i R \approx 0.53)$, these errors were ca. $0.71 \%$ and $0.82 \%$, respectively. For $N=512$ $(C i R \approx 1.07)$, the maximum phase estimation error was ca. $5.1 \cdot 10^{-2} \mathrm{rad}$ without the exponential 
component and ca. $8.3 \cdot 10^{-2}$ rad after the occurrence of this component, whereas, for $N=256$ $(C i R \approx 0.53)$, these errors were ca. $6.3 \cdot 10^{-2} \mathrm{rad}$ and $7.7 \cdot 10^{-2} \mathrm{rad}$, respectively. The results are more accurate and obtained quicker than those obtained with the methods presented in [28-31] (including the Least Mean Square Method - LMS), even when the signal frequency is estimated (not known a priori, as in [28-31]), which is necessary in practice.

The execution time for (20) and (23) is shorter than the execution time for the FFT procedure (Table 3 ) with $6.1 \%$ and $6.9 \%$ of the processor load, respectively. The pre-filter additionally involves the processor in $13.4 \%$, which does not significantly affect the total calculation time. The total load can be reduced by using a smaller number of samples $N$, a pre-filter with worse parameters or a processor with a higher clock rate frequency.

Table 3. The execution time of operations on TMS320C6713 in the implemented system.

\begin{tabular}{|c|c|c|c|c|c|c|}
\hline \multirow{2}{*}{ Part of the program } & \multicolumn{2}{|c|}{$\begin{array}{c}\text { Number } \\
\text { of cycles } T_{c}{ }^{1)}\end{array}$} & \multicolumn{2}{c|}{$\begin{array}{c}\text { Execution time } \\
\text { [ } \mu \mathrm{s}]\end{array}$} & \multicolumn{2}{c|}{$\begin{array}{c}\text { Processor load 2) } \\
{[\%]}\end{array}$} \\
\hline for $N=$ & 256 & 512 & 256 & 512 & 256 & 512 \\
\hline Prefiltering & 5050 & 5050 & 22.4 & 22.4 & 13.4 & 13.4 \\
\hline FFT algorithm & 4452 & 11590 & 19.8 & 51.5 & 11.9 & 30.8 \\
\hline$f_{1}$ estimation: [11] & 863 & 863 & 3.8 & 3.8 & 2.3 & 2.3 \\
\hline$A_{1}$ estimation: Eq. (20) & 2290 & 2290 & 10.2 & 10.2 & 6.1 & 6.1 \\
\hline$\varphi_{1}$ estimation: Eq. (23) & 2612 & 2612 & 11.6 & 11.6 & 6.9 & 6.9 \\
\hline Others & 4912 & 5559 & 21.8 & 24.7 & 13.1 & 14.8 \\
\hline Total & 20179 & 27964 & 89.6 & 124.2 & 53.7 & 74.3 \\
\hline
\end{tabular}

1) $T_{c}=(225 \mathrm{MHz})^{-1} \approx 4.4 \mathrm{~ns}(\mathrm{TMS} 320 \mathrm{C} 6713$ clock $)$.

2) Processor load is calculated as the ratio of "Execution time" to the period $4 T \approx 167 \mu \mathrm{s}$, where $T=1 / f_{\mathrm{s}} \approx 41.7 \mu \mathrm{s}$.

3) Other operations include: reading data from the $\mathrm{A} / \mathrm{D}$ converter, preparing the data buffer, storing data in memory, etc.

\section{Conclusions}

This paper presents a method of estimating the amplitude and phase of the grid signal. The method is an extension of the frequency estimation method presented in [11]. It uses the FFT procedure and maximum side-lobes windows of $H>1$ order. The possibility of using different windows from the used window family enables adjusting to specific problems of signal processing.

Computer simulations confirm correctness of the presented method. The accuracy of amplitude and phase estimation for a pure sinusoid depends on the measurement time $(\mathrm{CiR})$ and the number of samples $N$ collected during the measurement from the_A/D converter (Figs. 1,2) - the errors decrease when these values increase (dependency on $N$ is very strong - inversely proportional to $\mathrm{N}^{4}$ ). For the difficult case $C i R=0.1$ (the measurement time is only $10 \%$ of the grid signal period, i.e., $1.67 \mathrm{~ms}$ for $60 \mathrm{~Hz}$ or $2 \mathrm{~ms}$ for $50 \mathrm{~Hz}$ ) and $N=2048$, the amplitude and phase estimation errors are ca. $10^{-12}$ and ca. $10^{-12} \mathrm{rad}$, respectively, as shown in Figs. 1, 2. This means that, for the amplitude and phase of a pure sinusoid, their estimation errors, as well as the frequency estimation error [11], are negligible. However, in practice, the grid signal is heavily distorted by subharmonics, harmonics and noise. Moreover, the signal parameters may change over time. Adaptation of the algorithm to the parameter values changing in time does not exceed the $N T$ value (or equivalent $C i R$ ) (Figs. 7, 8), such as in the case of changing the frequency and its estimation [11].

The quality of the method for grid signal parameters estimation in the presence of noise is characterized by the ratio of the root square of the variance estimator (here, the $e M S E$ error) to $C R B$ bounds - the smaller the ratio, the better the method; the impossible limit is 1 . For the method presented in this paper, this ratio remains constant regardless of the noise power added 
to the test signal. For example, for $C i R=0.7$ this ratio is ca. 1.76 , whereas for $C i R=1.5$ it is ca. 5.25. An increase of $S N R$ value (a decrease of the noise power added to the signal) causes a decrease of the estimation errors and $C R B$ bounds (Figs. 3, 4). A significant source of noise is the quantization noise made by the $\mathrm{A} / \mathrm{D}$ converter, and, to maintain a high accuracy of the presented method, it is recommended to use the converter with the number of bits equal to $b$ and not less than 16 [32]. Even for accurate converters $(b \geq 16)$, the error caused by the quantization noise is greater than the method error for a pure sinusoid $[11,32]$.

The biggest impact on the estimation results is caused by subharmonics and harmonics, as demonstrated by the simulations in Section 5 and the experiment described in Section 6. Basing the method on the signal spectrum enables using the pre-filtration employing universal digital filters. In this paper, bandpass FIR filters were used (A and B filters in Section 5 and A filter in Section 6), but the task of choosing a filter type for different grid signal distortions is, according to the authors, worthy of a further study - the capabilities are large because of a wide variety of different types of digital filters, and the desired objective is to increase the filter accuracy and decrease the delay time brought by the filter.

Comparison with other methods based on the results obtained by other researchers [28-31], including the LMS method (which is known to be very accurate), shows that the method based on the interpolated DFT spectrum presented in the paper is a good alternative. It is also very important that the method uses the same spectrum points to estimate the amplitude, phase and frequency [11]. Therefore, the amplitude and phase estimation slightly increases the calculation time of signal parameters (Table 3 ).

The results show that the amplitude and phase estimation method presented in this paper, together with the frequency estimation method given in [11], can be successfully applied in control applications of renewable energy systems, especially photovoltaic systems. The research results of both simulations and implementation in a real-time DSP system confirm correctness of the developed methods. The research results of Sections 5 and 6 enable assessing the influence of individual harmonics in the signal on the parameter estimation accuracy and choosing the pre-filter parameters.

\section{References}

[1] Kabir, M.N., Mishra, Y., Ledwich, G., Dong, Z.Y., Wong, K.P. (2014). Coordinated Control of Grid-Connected Photovoltaic Reactive Power and Battery Energy Storage Systems to Improve the Voltage Profile of a Residential Distribution Feeder. IEEE Trans. on Ind. Inf., 10(2), 967-977.

[2] Rahim, N.A., Selvaraj, J., Solangi, K.H. (2013). Energy policy to promote photovoltaic generation. Renew. \& Sust. Energy Rev., 25, 44-58.

[3] Characteristics of the utility interface for photovoltaic (pv) systems. IEC 61727-2002.

[4] Measurement and assessment of power quality characteristics of grid connected wind turbines. IEC 61400-21.

[5] Eren, S., Pahlevaninezahad, M., Bakhshai, A., Jain, P.K. (2013). Composite Nonlinear Feedback Control and Stability Analysis of a Grid-Connected Voltage Source Inverter With LCL Filter. IEEE Trans. on Ind. Elec., 60(11), 5059-5074.

[6] Kanieski, J., Cardoso, R., Pinheiro, H., Grundling, H.A. (2013). Kalman Filter-Based Control System for Power Quality Conditioning Devices. IEEE Trans. on Ind. Elec., 60(11), 5214-5227.

[7] Vazquez, S., Sanchez, J.A., Reyes, M.R., Leon, J.I., Carrasco, J.M. (2014). Adaptive Vectorial Filter for Grid Synchronization of Power Converters Under Unbalanced and/or Distorted Grid Conditions. IEEE Trans. on Ind. Elec., 61(3), 1355-1367.

[8] Shahbazi, M., Poure, P., Saadate, S., Zolghadri, M.R. (2013). FPGA-Based Fast Detection With Reduced Sensor Count for a Fault-Tolerant Three-Phase Converter. IEEE Trans. on Ind. Inf., 9(3), 1343-1350.

[9] Feola, L., Langella, R., Testa, A. (2013). On the Effects of Unbalances, Harmonics and Interharmonics on PLL Systems. IEEE Trans. on Instr. and Meas., 62(9), 2399-2409. 
[10] Yang, Y., Zhou, K., Cheng, M. (2013). Phase Compensation Resonant Controller for PWM Converters. IEEE Trans. on Ind. Inf., 9(2), 957-964.

[11] Borkowski, J., Kania, D., Mroczka, J. (2014). Interpolated DFT-Based Fast and Accurate Frequency Estimation for the Control of Power. IEEE Trans. on Ind. Elec., 61(12), 7026-7034.

[12] Štremfelj, J., Agrež, D. (2013). Nonparametric estimation of power quantities in the frequency domain using RifeVincent windows. IEEE Trans. on Instr. and Meas., 62(8), 2171-2184.

[13] Belega, D., Petri, D., Dallet, D. (2014). Frequency estimation of a sinusoidal signal via a three-point interpolated DFT method with image component interference rejection capability. Digital Signal Processing, 24(1), $162-169$.

[14] Chen, C.I. (2013). A Phasor Estimator for Synchronization Between Power Grid and Distributed Generation System. IEEE Trans. on Ind. Elec., 60(8), 3248-3255.

[15] Jain, S.K., Singh, S.N. (2013). Fast Harmonic Estimation of Stationary and Time-Varying Signals Using EAAWNN. IEEE Trans. on Instr. and Meas., 62(2), 335-343.

[16] Huang, X., Guo, Y.J. (2010). MSE lower bounds for phase estimation based on overlapped Gaussian distribution. Intern. Symp. on Commun. and Inform. Techn.

[17] Fu, H., Kam, P.Y. (2013). Phase-Based, Time-Domain Estimation of the Frequency and Phase of a Single Sinusoid in AWGN - The role and Applications of the Additive Observation Phase Noise Model. IEEE Trans. on. Inf. Theory, 59(5), 3175-3188.

[18] Yamada, T. (2013). High-Accuracy Estimations of Frequency, Amplitude, and Phase With a Modified DFT for Asynchronous Sampling. IEEE Trans. on Instr. and Meas., 62(6), 1428-1435.

[19] Dash, P.K., Hasan, S. (2011). A Fast Recursive Algorithm for the Estimation of Frequency, Amplitude, and Phase of Noisy Sinusoid. IEEE Trans. on Ind. Elec., 58(10), 4847-4856.

[20] Belega, D., Macii, D., Petri, D. (2014). Fast Synchrophasor Estimation by Means of Frequency-Domain and TimeDomain Algorithms. IEEE Trans. on Instr. and Meas., 63(2), 388-401.

[21] Rife, D.C., Vincent, G.A. (1970). Use of the Discrete Fourier Transform in the Measurement of Frequencies and Levels of Tones. Bell Syst. Tech. Journal, 49, 197-228.

[22] Nuttall, A.H. (1981). Some Windows with Very Good Sidelobe Behavior. IEEE Trans. on Acous., Sp. and Sig. Proc., 29(1), 84-91.

[23] Belega, D., Dallet, D. (2008). Frequency estimation via weighted multipoint interpolated DFT. IET Scien., Meas. and Tech., 2(1), 1-8.

[24] Rife, D.C., Boorstyn, R.R. (1974). Single-Tone Parameter Estimation from Discrete-Time Observations. IEEE Trans. on Inform. Theory, 20(5).

[25] IEEE Standard for Synchrophasors for Power Systems. IEEE Std. C37.118-2005.

[26] Chen, C.I. (2013). A Phasor Estimator for Synchronization Between Power Grid and Distributed Generation System. IEEE Trans. on Ind. Elec., 60(8), 3248-3255.

[27] Voltage characteristics of electricity supplied by public electricity networks. EN 50160:2010.

[28] Alhaj, H.M.M., Nor, N.M., Asirvadam, V.S., Abdullah, M.F. (2014). Power system harmonics estimation using LMS, LMF and LMS/LMF. Intern. Conf. on Intel. and Adv. Syst.

[29] Subudhi, B., Ray, P.K. (2009). Estimation of Power System Harmonics Using Hybrid RLS-Adaline and KFAdaline Algorithms. IEEE Region 10 Conference TENCON.

[30] Alhaj, H.M.M., Nor, N.M., Asirvadam, V.S., Abdullah, M.F. (2013). Power System Harmonics Estimation using Sliding Window Based LMS. IEEE Intern. Conf. on Signal and Image Proc. Applications.

[31] Ray, P.K., Subudhi, B. (2012). Ensemble-Kalman-Filter-Based Power System Harmonic Estimation. IEEE Trans. on Instr. and Meas., 61(12), 3216-3224.

[32] Borkowski, J., Kania, D., Mroczka, J. (2014). Influence of A/D Quantization in Interpolated-DFT-Based System of Power Control with Small Delay. Metrol. Meas. Syst., 21(3), 423-432. 